\title{
TERWILLIGER IN THE DEPARTMENT AND UNIVERSITY
}

\author{
H.A. Neal \\ University of Michigan, Ann Arbor, MI 48109-1120
}

In the many presentations that have preceded mine, there has been much discussion of Kent's accomplishments as a scientist. My special task in this particular session is to comment on his service to the department and to the university, in his role as Associate Chair for Research and Facilities.

As a physicist, Kent would be very unhappy for us to have a discourse on assessments, if we had not specified beforehand by what standards the assessments were to be made. So let us digress for a few minutes, to discuss what makes an outstanding administrator. He or she should be a person who listens to the needs and aspirations of those being served, and exerts every effort to facilitate both the short term and long term goals of colleagues. An administrator should be a person of integrity and be sensitive and fair. The person should recognize the nature of the environment in which he or she works, and master the language - budgetary and otherwise - of the various units with which he or she must interact.

An effective administrator should have a vision as to where the enterprise is going and know what must be done to get there. He or she must have extraordinary patience, when patience is called for, and yet be ready to act without delay, in instances where that is appropriate - even if such action involves some risk. The ideal administrator must have a keen sense of quality that will permit him or her to make sound priority decisions in an environment where there is never enough resources to meet all of the requests being made. An administrator must be able to work harmoniously with the wide spectrum of individuals, even though each of them have their own personalities and agendas.

These are just a few of the metrics that one can use to measure the success of an administrator, and, if we apply these measures to Kent's years of service, it is clear that we were extremely fortunate to have benefited over the years from his service as associate chair.

In the role of Associate Chair, Kent managed a variety of tasks, ranging from arranging matching funds for sponsored research for our faculty, providing direct research funding support, providing oversight of the instrument shop, the student shop, handling space assignments, and participating in departmental research administration in myriads of ways. Each of these areas is a potential land-mine, if managed improperly. And that these operations proceeded so smoothly under Kent's purview is indeed a result of his very special talents. Over the course of his career, Kent made tremendous contributions to the life of this department, both as a physicist and as an administrator. 


\section{Terwilliger in the Department and University}

As Kent observed the size of our Department grow from 30 faculty in the mid-50's to 58 now, he also observed how the field itself was changing in substance and technique. Nuclear physics spawned particle physics and solid state physics became condensed matter physics. Lasers revolutionized optical and spectroscopic physics; large new detectors opened new realms of observation of astrophysical phenomenon, as well as of high energy physics phenomena. Michigan has played important roles in a number of these changes, and Kent was an integral part of this evolution.

Our department is active in a number of areas, including Nuclear Physics, Experimental Astrophysics, High Energy Physics, Condensed Matter Physics, Biophysics, Musical Acoustics, Atomic Physics, Precision Measurements, Optical Physics, and Energy Resources. The traditions of excellence here are unsurpassed, including being the academic home of physicists who discovered electron spin and proton spin; the inventors of the $\mathrm{g}-2$ techniques for measuring the electron and positron $\mathrm{g}$-factors; the inventor of the bubble chamber; the discoverers of non-linear optical effects and of techniques that now make fiber optics a reality. On a more recent timescale, I remind you of the work of several of our faculty in observing neutrinos from the Supernova 1987a in the renowned IMB experiment conducted in salt mines near Cleveland. This is the Department which Kent called home - and the Department that is proud to count Kent's work, such as the Terwilliger quadrupole development, among its list of unique contributions to the advancement of physics.

Kent's area of research was high energy physics. Our Department has one of the strongest high energy physics programs in the country. Indeed, in a recent study it was noted that the if one were to rank universities (without national laboratories) by total NSF and DOE expenditures for high energy research, Michigan would be third in the country. Our faculty are engaged in high energy research at Brookhaven, Fermilab, Stanford, CERN, and in Russia. Moreover, our faculty have been active in supporting the SSC project, both through working with the public and in working on various technical problems. As in other areas, we are adding new outstanding faculty in high energy physics, and have every expectation that the vitality of the Michigan high energy physics program, to which Kent contributed so much, will be preserved in the years ahead.

To build a first class research program of the sort that we have in this department requires extensive technical support. Remaining at the frontier of physics requires skilled technicians, with proper tools, who can work closely with the faculty in bringing experimental ideas to realization. Michigan has a long tradition of excellence in instrument-making, a tradition which Kent clearly helped to perpetuate. He oversaw important developments in the instrument shop. Indeed, his long-standing concern about the Instrument Shop is exemplified by his concluding in recent months the purchase of an expensive CNC milling ma- 
chine for our shop from his hospital bed. Without this type of attention over the years, it would have been impossible for the department to produce the mechanical and electronic components which have made it an important leader in frontier experimental physics.

A first class research department requires at least a certain minimum amount of quality space. Though we have not always been blessed in this regard, I hope you will note that this is changing. Kent did much to help in launching us on our present program of space expansion. Our plans include a complete renovation of Randall Laboratory, the renovation and occupancy of considerable space in West Engineering, and the construction of a new laboratory building. The University has committed itself to cover the renovation costs, and indeed these projects are underway. Our request for funding the new building has already cleared several milestones in Lansing, and we are optimistic that this project will move forward expeditiously. Kent was enthusiastically involved in all aspects of the development of these aggressive plans.

In summary, Kent Terwilliger was a beloved colleague, an outstanding physicist, and a talented administrator. He had a lasting impact on building a department that is one of the premier research centers in the physics world.

I wish to record for the proceedings the formal document from the Regents of the University received by the department in connection with Kent's passing. It makes mention of several items which many of you have expressed during the past two days. It is an acknowledgment by the faculty of the University of the loss of one of its highly valued members.

I also wish to call to your attention to the memorial in the June '89 issue of the CERN Courier. It was heartening to see the acknowledgment of Kent's role in development of the Terwilliger quadrupoles and his broad contributions to our field.

In closing, I would like to express my personal thanks to each of you who have helped us to honor the memory of Kent and to celebrate his life. 


\title{
THE UNIVERSITY OF MICHIGAN \\ REGENTS COMMUNICATION
}

\author{
ACTION REQUEST
}

Subject:

Report of Faculty Death

Action Requested: Adoption of Memorial

The Regents of the University acknowledge with profound sadness the death on February 23, 1989 of Kent Melville Terwilliger, professor of physics and associate chair for research and facilities in the Department of Physics.

A pioneer in the field of experimental high energy physics, Professor Terwilliger was inventor of the "Terwilliger quadrupole" -- a magnet system used in colliding high energy particle beams; indeed, he was a co-author of the first article demonstrating the feasibility of colliding particle beams, in 1956. In recent years he became one of the world's leading experts in the field of accelerated polarized proton beams.

Professor Terwilliger was born in San Jose, California in 1924. He received his B.S. degree from the California Institute of Technology in 1949 and his Ph.D. degree in physics from the University of California at Berkeley in 1952. He joined the University of Michigan Department of Physics in the fall of 1952.

Kent served on a number of national high-energy physics committees and panels: he was a member of the high energy physics advisory panel of the Atomic Energy Commission from 1968-71 and chair of the high energy physics users group at Argonne National Laboratory from 1962-64 and a Trustee of AUA, which manages Argonne National Laboratory. On these and other committees, Kent was much esteemed for his precise thought and astute insights. Kent was a recipient of a Guggenheim Fellowship and was a member of the American Physical Society and the American Association for the Advancement of Science.

In the Department of Physics, Kent was highly valued as a teacher and collaborator and warmly regarded as a friend. A modest and self-effacing man, quiet and reserved, he had the rare ability to pinpoint the crux of a complex research problem. Always generous and supportive of the work of others, he filled his role as associate chair for research with distinction; the faculty felt very welcome to bring their problems and questions to him.

Kent was a physicist in mind and in spirit, with a deep love and respect for the physical world in all its complexity. He was daring as a scientist -- a fact that his modesty as a man occasionally concealed. His rigorous scientific honesty and careful research provided a standard which inspired other scientists locally, nationally, and indeed, internationally.

Kent has left an enduring mark on his students and colleagues, both through his accomplishments as a physicist and his qualities as a person. He will be long remembered as an unusually dedicated physics researcher who displayed dignity and warmth in his efforts to study the universe, with no apparent interest in personal recognition. As we mourn the loss of this great scholar and teacher, our condolences go to his wife Doris and his sons Steven, Paul, Thomas, and John.

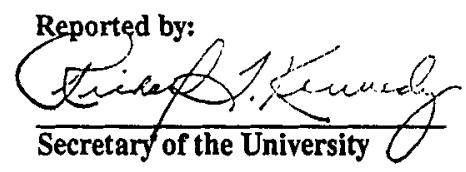




\section{Kent M. Terwilliger Doctoral Students; Year of Ph.D. and Thesis Title}

Lavon L. Yoder (1963) "Associated Production in $1.5 \mathrm{BeV} / \mathrm{c} \pi^{-}-p$ Interactions and Kaon Lifetimes".

Larenzo J. Curtis (1963) (co-chair) "An Investigation of the (Y*) PionHyperon Resonance in $(1.5 \mathrm{BeV} / \mathrm{c}) \pi^{-}-p$ Interactions".

Robert W. Anthony (1971) "Recoil Inelastic Pion Spectra Resulting from 2.15 to $6.00 \mathrm{GeV} / \mathrm{c}$ Pi-minus Proton Interactions".

James E. Rice (1971) "Study of the Reaction $\pi^{-} p \rightarrow \pi^{ \pm} N^{*-}(1236)$ near $180^{\circ}$ from 2.15 to $6.00 \mathrm{GeV} / \mathrm{c}^{\prime \prime}$.

Nikoletsa Merminga (1989) (co-chair) "A Study of Nonlinear Dynamics in the Fermilab Tevatron". 
KENT M. TERWILLIGER

Bibliography of Journal Publications and Conference Proceedings

\section{PUBLICATIONS}

1951

1. Relative Photoneutron Yields from $330 \mathrm{MeV}$ Bremsstrahlung, Phys. Rev., 82, 820-822 (1951) (with L.W. Jones and W.N. Jarmie).

1952

2. Comparison of Total Photoneutron Yields from 160 to $320 \mathrm{MeV}$ Bremsstrahlung, Phys. Rev., 85, 689 (1952) (with L.W. Jones).

1953

3. Photoneutron Production Excitation Functions to $320 \mathrm{MeV}$, Phys. Rev., 91, 699-707 (1953) (with L.W. Jones).

1955

4. Betatron Oscillations in the Synchrotron, Rev. Sci. Instrum., 26, 555-556 (1955) (with C.L. Hammer and R.W. Pidd).

1956

5. Attainment of Very High Energy by Means of Intersecting Beams of Particles, Phys. Rev., 102, 590 (1956) (with 9 others).

6. Experimental Test of the Fixed Field Alternating Gradient Principle of Particle Accelerator Design, Rev. Sci. Instrum., 27, 651 (1956) (with L.W. Jones and R.O. Haxby).

7. Fixed Field Alternating-Gradient Particle Accelerators, Phys. Rev., 103, 1837-1859 (1956) (with 4 others). (Also translated and printed in Russian Review journal "Progress in Physical Science", 61, 613-652 (1957).

\section{7}

8. Electron Model Fixed Field Alternating Gradient Accelerator, Rev. Sci. Instrum., 28, 403-420 (1957) (with 4 others).

9. Beam Stacking Experiments in an Electron Model FFAG Accelerator, Rev. Sci. Instrum., 28, 987-997 (1957) (with L.W. Jones and C.H. Pruett).

\section{$\underline{1962}$}

10. High Energy Particle Accelerators, School Sci. Math., 12, 189-196 (1962). 


\section{3}

11. Production of the $1385 \mathrm{MeV}\left(\mathrm{Y}_{1}^{*}\right)^{\circ}$ in $1.5 \mathrm{BeV} / \mathrm{c} \pi^{-}$-p Collisions, Phys. Rev., 132, 1771-1778 (1963) (with L.J. Curtis, C.T. Coffin and D.I. Meyer).

12. $\lambda \mathrm{K}^{\circ}$ and $\Sigma^{\circ} \mathrm{K}^{\circ}$ Production in $1.5 \mathrm{BeV} / \mathrm{c} \pi^{-} \mathrm{p}$ Interactions, Phys. Rev. 132, 1778-1781 (1963) (with L.L. Yoder, C.T. Coffin, and D.I. Meyer).

\section{5}

13. $\pi^{-}+\mathrm{p}$ Elastic Scattering from 2.5 to $6 \mathrm{GeV} / \mathrm{c}$, Phys. Rev. Lett., $\underline{15}, 838-841$ (1965) (with others).

\section{6}

14. $\pi^{+}$p Elastic Differential Cross Sections from 2.3 to $4 \mathrm{GeV} / \mathrm{c}$, Phys. Rev. Lett., 17, 458-461 (1966) (with others).

15. $\mathrm{k}^{-} \mathrm{p}$ Charge Exchange at 5,7 , and $12 \mathrm{GeV} / \mathrm{c}$, Phys. Lett., $\underline{23}$, 396-400 (1966) (with others).

\section{7}

16. Elastic Differential Cross Sections for $\pi^{ \pm}+p$ Scattering from 2.3 to $6.0 \mathrm{BeV} / \mathrm{c}$, Phys. Rev., 159 , 1169-1175 (1967) (with others).

17. Observation of Resonances in the $\mathrm{K}_{1} \mathrm{~K}_{1}$ System, Phys. Lett., 25B, 357-361 (1967) (with others).

\section{$\underline{1968}$}

18. $\pi^{-} \mathrm{p}$ Backward Elastic and Inelastic Scattering at $2.15 \mathrm{GeV} / \mathrm{c}$, Phys. Rev. Lett., 21, 1605-1608 (1968) (with others).

\section{1}

19. Backward Pion Production from $\pi^{-}$p Interactions, Phys. Rev. Lett., 26, 38-41 (1971) (with others).

20. Study of the Reaction $\pi^{-} p \rightarrow \pi^{+} \triangle(1236)$-in the Backward Direction at 2.15 to $6 \mathrm{GeV} / \mathrm{c}$, Phys. Rev. Lett., 27, 687-689 (1971) (with others).

\section{2}

21. $\pi^{-} \mathrm{p}$ Elastic Scattering Near $180^{\circ}$ from 2.15 to $6 \mathrm{GeV} / \mathrm{c}$, Phys. Rev., 6D, 740-746 (1972) (with others).

22. The Distribution in Transverse Momentum of $5 \mathrm{GeV} / \mathrm{c}$ Secondaries Produced at $53 \mathrm{GeV}$ in the Center of Mass, Phys. Lett., $\underline{42 \mathrm{~B}, 279-}$ 282 (1972) (with others).

\section{3}

23. $\rho$ Production and Decay in the Reaction $\pi^{-} \mathrm{p} \rightarrow \pi^{-} \pi^{\circ} \mathrm{p}$ at 5 $\mathrm{GeV} / \mathrm{c}$, Phys. Rev., D7, 637-660 (1973) (with 7 others). 
24. Longitudinal-Momentum Distributions for Positive Particles Produced at Small Angles in Proton-Proton Collisions at a C.M. Energy of $44.6 \mathrm{GeV}$, Nucl. Phys., B51, 388-396 (1973) (with 15 others).

\section{4}

25. Simultaneous Measurement of 2 and 3 Spins in Proton Proton Elastic Scattering at $6 \mathrm{GeV} / \mathrm{c}$, Phys. Lett., 52B, 243-246 (1974) (with 9 others).

\section{$\underline{1975}$}

26. New Measurement of $\sigma_{\text {TOT }}$ in Proton-Proton Scattering in Pure

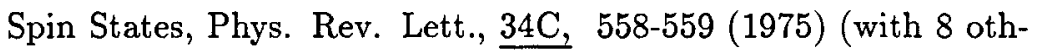
ers).

\section{6}

27. Measurement of Proton Proton Elastic Scattering in Pure Initial Spin States at $11.75 \mathrm{GeV} / \mathrm{c}$, Phys. Lett., 63B, 239-244 (1976) (with 8 others).

\section{7}

28. Proton Elastic Scattering at $6.0 \mathrm{GeV} / \mathrm{c}$ with Three Spins Measured, Phys. Rev., D15, 604-608 (1977) (with 9 others).

29. High- $P_{\perp}^{2}$ p-p Elastic Scattering in Pure Initial Spin States, Phys. Rev., D16, 549-552 (1977) (with 10 others).

30. Spin-spin Interactions in High- $P_{\perp}^{2}$ Elastic p-p Scattering, Phys. Rev. Lett., 39, 733-736 (1977) (with 11 others).

1978

31. Measurement of Proton-Proton Elastic Scattering at $6 \mathrm{GeV} / \mathrm{c}$ in Polarized Initial and Final Spin States, Phys. Rev., D17, 24-41 (1978) (with 9 others).

32. Energy Dependence of Spin-Spin Forces in $90^{\circ} \mathrm{cm}$ Elastic p-p Scattering, Phys. Lett., 74B, 273-276 (1978) (with 9 others).

33. Spin Dependence of High-P $\mathrm{P}_{\perp}^{2}$ Elastic p-p Scattering, Phys. Rev. Lett., 41, 1257-1259 (1978) (with 10 others).

\section{9}

34. Spin-Spin Forces in $6 \mathrm{GeV} / \mathrm{c}$ Neutron-Proton Elastic Scattering,

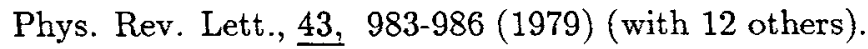

1981

35. Energy Dependence of Spin-Spin Effects in p-p Elastic Scattering at $90^{\circ} \mathrm{cm}$, Phys. Rev., D23, 600-603 (1981) (with 15 others). 
1982

36. Measurements of Spin Parameters in p-p Elastic Scattering at 6 $\mathrm{GeV} /$ c. Phys. Rev., D26, 550-556 (1982) (with 14 others).

1983

37. Spin Analyzing Power in p-p Elastic Scattering at $28 \mathrm{GeV} / \mathrm{c}$, Phys. Rev. Lett., 50, 802-806 (1983) (with 15 others).

38. Large- $\mathrm{P}_{\perp}^{2}$ Spin Effects in $\mathrm{p}+\mathrm{p} \rightarrow \mathrm{p}+\mathrm{p}$, Phys. Rev. Lett., $\underline{51}$ 2359-2361 (1983) (with 13 others).

1985

39. Measurement of $\mathrm{p}_{\uparrow}+\mathrm{p}_{\uparrow} \rightarrow \mathrm{p}+\mathrm{p}$ with a $16.5 \mathrm{GeV} / \mathrm{c}$ Polarized Proton Beam, Phys. Rev., D31, Rapid Communications, 30173019 (1985) (with 16 others).

40. Measurement of the Analyzing Power for $p+p_{\uparrow} \rightarrow p+p$ at $\mathrm{P}_{\perp}^{2}=6.5(\mathrm{GeV} / \mathrm{c})^{2}$, Phys. Rev., D32, Rapid Communications, 3070-3072 (1985) (with 16 others).

$\underline{1986}$

41. Energy Dependence of Spin Effects in $\mathrm{p}_{\uparrow} \mathrm{p}_{\uparrow} \rightarrow \mathrm{p}+\mathrm{p}$, Phys. Rev. Lett., $57,507-510$ (1986) (with 17 others).

1988

42. Measurement of Spin Effects in $\mathrm{p}_{\uparrow}+\mathrm{p}_{\uparrow} \rightarrow \mathrm{p}+\mathrm{p}$ at $18.5 \mathrm{GeV} / \mathrm{c}$, Phys. Rev. Lett., 60, 2351-2354 (1988) (with 15 others).

1989

43. The Acceleration of Polarized Protons to $22 \mathrm{GeV} / \mathrm{c}$ and the Measurement of Spin Effects in $p_{\uparrow}+p_{\uparrow} \rightarrow p+p$, Phys. Rev., (1989) (with 42 others).

44. First Test of the Siberian Snake Magnet Arrangement to Overcome Depolarizing Resonances in a Circular Accelerator, Phys. Rev. Lett. $\underline{63}$, $1137-1140$ (1989) (with 18 others).

\section{CONFERENCE PROCEEDINGS}

1956

45. Fixed Field Alternating Gradient Particle Accelerators, Proc. of CERN Symposium on High Energy Accelerators and Pion Physics (CERN, Geneva, 1956), 32-35 (with K.R. Symon and others).

46. A Small Model Fixed Field Alternating Gradient Radial Sector Accelerator, Proc. of CERN Symposium on High Energy Accelerators and Pion Physics (CERN, Geneva, 1956), 359-365 (with L.W. Jones). 
47. Beam Extraction from FFAG Synchrotrons, Proc. of International Conf. on High Energy Accelerators (CERN, Geneva, 1959), 48-53 (with L.W. Jones).

48. Achieving Higher Beam Densities by Superposing Equilibrium Orbits, Proc. of International Conf. on High Energy Accelerators (CERN, Geneva, 1959), 53-57.

49. Comparison of Experimental Results with the Theory of Radiofrequency Acceleration Processes in FFAG Accelerators, Proc. of International Conf. on High Energy Accelerators (CERN, Geneva, 1959), 58-70 (with L.W. Jones, C.H. Pruett, and K.R. Symon).

50. Orbit Dynamics for a Four Spiral Sector Cyclotron, Proc. of Conf. on Sector-Focused Cyclotrons, Pub. 656, Nat. Acad. of Sci., Nat. Res. Council (1959), 73-75.

\section{0}

51. Thin Foil Discharge Chambers, Proc. of International Conf. on Instrumentation for High Energy Physics (LRL, Berkeley, 1960), 276 (with D.I. Meyer).

1961

52. A 14" x 14" x 7" Thin Plate Spark Chamber, Proc. 1961 Spark Chamber Symposium, Rev. Sci. Instrum. 32, 512-515 (1961) (with D.I. Meyer).

1962

53. Mass Spectrum of the $1385 \mathrm{MeV} \lambda^{\circ}+\pi^{\circ}$ Resonance from $1.5 \mathrm{GeV} / \mathrm{c}$ $\pi$-Mesons on Protons, Proc. of the 1962 International Conference on High Energy Physics at CERN, 327-329 (with C.T. Coffin, L.J. Curtis and D.I. Meyer).

\section{3}

54. Use of Relative Ionization for Particle Identification in Multitrack Spark Chamber Pictures, Proc. 1962 Conf. on Instrumentation for High Energy Physics, Nucl. Instrum. Methods, 20, 156-160 (1963) (with C.T. Coffin, L.J. Curtis and D.I. Meyer).

\section{$\underline{1966}$}

55. Conventional and Digitized Spark Chambers, invited rapporteur paper, Proc. of the 1966 International Conf. on Instrumentation for High Energy Physics (SLAC, Stanford, 1966), 389-408.

1978

56. Spin Measurements in Hadronic High Momentum Transfer Scattering, invited review talk, Proc. of the III International Symposium on High Energy Physics with Polarized Beams and Targets, (Argonne, 1978), G.H. Thomas, ed., (AIP, New York), 521-541. 


\section{9}

57. The Possibility of Acceleration of Polarized Protons in the Brookhaven AGS. IEEE Transactions on Nuclear Science, NS-26, 3202-3205 (1979) (with 8 others).

58. Spin Dependence in High- $\mathrm{P}_{\perp}^{2}$ Elastic pp and np Scattering. Presented by K.M. Terwilliger, Proc. International EPS Conference on High Energy Physics, (Geneva, 1979), 2, 667-669 (1979) (with 18 others).

59. Early Strong Interaction Counter Experiments, invited talk, Symposium on the History of the ZGS, (Argonne National Laboratory, 1979), J.S. Day, A.D. Krisch, L.G. Ratner, eds., AIP Conf. Proc., 60, 70-81 (AIP, New York)

\section{0}

60. Energy Dependence of Spin-Spin Effects in pp Elastic Scattering at $90^{\circ} \mathrm{cm}$, Presented by K.M. Terwilliger., Proc. of Symposium on High Energy Physics with Polarized Beams and Polarized Targets, (Lausanne, 1980), C. Joseph and J. Soffer, eds., (Berkhauser Verlag, 1981), p. 545-548 (with 15 others).

61. Spin Effects in Nucleon-Nucleon Elastic Scattering, Presented by K.M. Terwilliger, Proc. of the XX International Conference on High Energy Physics, (Madison, Wis. 1980), AIP Conf. Proc., 68, 54-55 (1980) (with 17 others).

\section{1}

62. Acceleration of Polarized Protons in the Brookhaven AGS, Presented poster session, 1981 Particle Accelerator Conference, Washington, DC, IEEE Trans. on Nuc. Sci., NS-28, 2031-2033 (1981) (with 12 others).

\section{2}

63. Summary Report, Workshop on Acceleration of Polarized Protons, 5th International Symposium on High Energy Spin Physics-1982, Brookhaven National Laboratory, 450-453 (AIP 95, New York, 1983) (with Y.Y. Lee).

\section{3}

64. Polarimeters for the AGS Polarized Proton Beam, 1983 Particle Accelerator Conference, Sante Fe, IEEE Trans. on Nucl. Sci. NS30, 2176-2178, 1983 (with 14 others).

65. Polarized Protons in the SSC, Proceedings of the Workshop on Accelerator Physics Issues for a Superconducting Super Collider, Ann Arbor, December 1983 (UM HE 84-1) (1984) App. 1, 7, pp. 60-62 (with 8 others). 
1984

66. Analyzing Power Measurements for p-p Elastic Scattering at High$\mathrm{P}_{\perp}^{2}$, Proceedings of the Conference on Intersections Between Particle and Nuclear Physics, Steamboat Springs, (Colorado, 1984), AIP Conference Proceedings, 123, 1123-1125 (1984) (with 17 others).

\section{5}

67. Depolarizing "Beat" Resonances in the Brookhaven AGS, in Proceedings of 1985 Particle Accelerator Conference, (Vancouver), K.M. Terwilliger, A.D. Krisch, E.D. Courant, and L.G. Ratner eds., IEEE Trans. on Nuclear Science, Vol. NS-32, 2635-2637 (1985).

68. Commissioning the Polarized Beam in the AGS, in Proceedings of 1985 Particle Accelerator Conference, (Vancouver), IEEE Trans. on Nuclear Science, Vol. NS-32, 1656-1658 (1985) (with 16 others).

69. Analyzing Power Measurements for p-p Elastic Scattering at High$\mathrm{P}_{\perp}^{2}$, Proceedings of 1984 Marseille Symposium on High Energy Spin Physics, J. Soffer, ed., Journal de Physique, Colloque C2, Sup. $n^{\circ} 2$, Tome 46, p. C2-303-305 (1985) (with 17 others).

70. Round Table Discussion, Workshop on Polarized Beams at SSC, (Ann Arbor, Michigan, 1985), A.D. Krisch, A.M.T. Lin, and O. Chamberlain, eds., AIP Conf. Proc., 145, 130-131 (AIP, New York, 1986).

\section{7}

71. Polarized Proton Beams at the AGS, invited paper, IEEE Proc. of the 1987 Particle Accelerator Conf., (Washington, DC), p. 809-813 (March 1987).

\section{8}

72. Measurement of Spin Effects in pp Elastic Scattering Using the Polarized Beam at the AGS, Presented at the XXIV International Conference on High Energy Physics (Munich, August 1988), (with 15 others), to be published.

73. Hadron Beams Session--Summary, Presented at the Symposium on High Energy Spin Physics (Minneapolis, MN 1988), to be published. 